\title{
Analysis and Selection of Features for the Fingerprint Vitality Detection
}

\author{
Pietro Coli, Gian Luca Marcialis, and Fabio Roli \\ Department of Electrical and Electronic Engineering - University of Cagliari \\ Piazza d'Armi - I-09123 Cagliari - Italy \\ \{pietro.coli, marcialis, roli\}@diee.unica.it
}

\begin{abstract}
Although fingerprint verification systems have attained a good performance, researchers recently pointed out their weakness under fraudulent attacks by fake fingers. In fact, the acquisition sensor can be deceived by fake fingerprints created with liquid silicon rubber. Among the solutions to this problem, the software-based ones are the cheapest and less intrusive. They use feature vectors made up of measures extracted from one or multiple impressions (static measures) or multiple frames (dynamic measures) of the same finger in order to distinguish live and fake fingers. In this paper, we jointly use both static and dynamic features and report an experimental investigation aimed to compare them and select the most effective ones.
\end{abstract}

\section{Introduction}

Fingerprint matching algorithms are widely used for automatic personal verification [1]. Although the fingerprint verification systems have shown a good degree of accuracy, their weak point is the acquisition sensor, which can be of optical or solidstate type [1]. It has been shown by Matsumoto et al. that commonly used sensors can be deceived by submitting a "gummy" finger, made up of liquid silicon rubber and similar materials [2]. The image produced by this kind of fingers is processed as well as a "live" image.

Although reproducing fingerprint is not simple, the academic and commercial interest on spoof attempts is increasing. In order to prevent the fraudulent attempts by fake fingers, several solutions have been proposed. Most of them are based on the use of additional hardware, embedded in the sensor, which can detect the "vitality" of the finger, e.g., through the heartbeat detection.

A novel approach to the vitality detection of fingerprints has been proposed in [35]. It is based on the extraction of features which can discriminate between live and fake fingerprints by using the images acquired by the sensor. These software-based solutions are obviously less intrusive and cheaper than the hardware-based ones. So far, the state-of-the-art consists of two main approaches: the first one is based on the dynamic measure of some "intrinsic" features [3-4], while the second one uses relative measures between an unknown fingerprint image and another one which is known to be "live" [5]. The search of some physiologic or physic characteristics of the fingerprint, in order to derive some vitality measures, follows the observation that 
the "vitality" is a property of the fingertip and not of the fingerprint. Therefore, the main features considered so far are based on the perspiration of the skin through the pores and the "deformation" properties of the skin. To measure the first kind of features a temporal analysis of the fingerprint was adopted [3-4], whilst a static analysis was adopted for the second kind [5].

So far, static and dynamic features have been used separately. However, it is reasonable to argue that both features provide discriminant information about live and fake fingers. Accordingly, their joint contribution should be investigated. This is the purpose of this paper, which reports an experimental comparison aimed to select the most discriminant subsets of features for optimising the performance of automatic fingerprint vitality detection systems.

Section 2 presents the features we used for our experiments. Section 3 describes the used data set and the experiments performed for the feature selection process. Section 4 draws some preliminary conclusions.

\section{Static and Dynamic Features for the Fingerprint Vitality Detection}

The vitality detection by software-based solutions starts from the measure of some features extracted from the fingerprint image acquired by standard acquisition sensors (e.g., optical or capacitive [1]).

At present, the main approaches proposed in literature to perform vitality detection are based on the study of the skin perspiration through the pores and the elastic properties of the skin.

For the first kind of measures, the user hold his finger on the scanner surface, and the biometric system acquires different frames in the time. Features are extracted according to the differences among these frames.

For the second kind of measures, the vitality information is given by the different elastic response which is hypotesized to coming from true and fake fingers. In order to derive this type of information the user has to repeat the acquisition process on the scanner surface.

In the following, we refer to the features derived from these approaches by the terms "dynamic" and "static". In fact, to measure the first kind of features the "dynamic" acquisition of multiple frames of the same finger is required, whilst the "static" acquisition of multiple impressions of the same finger is required for the second kind.

The following sections describe the features we adopted for our experiments.

\subsection{Static Features}

The use of elastic measures has been adopted in previous works in order to correct the non-linear deformations introduced during the acquisition stage, and so improving the alignment between different impressions of the same fingerprint [6].

Recently, an elastic model has been adopted into a vitality detection system [5]. In this work Chen et al. compute an elastic model to study the different deformation of a fake fingerprint from a live one. They have observed that the contact of the fingertip with a plane surface involves an elastic deformation: the flow of papillary ridges 
changes from a 3D to a 2D-pattern. Through the mathematical model proposed in [6], Chen et al. showed that it is possible to study the elastic behaviour of a live and a fake finger.

The first step for the computation of this static measure is the extraction of a fixed and ordered set of minutiae from the fingerprints. For the purpose of this work we manually extracted the minutiae in order to eliminate all possible error sources. From a fingerprint $k$ we manually extracted 20 minutiae $M^{k}=\left(m^{k}{ }_{1}, m_{2}^{k}, \ldots m_{20}^{k}\right)$.

Then, starting from a couple of set of minutiae extracted from two fingerprints, namely, a "template" fingerprint and an input impression of the same subject, $M^{t}=\left\{m_{1}^{t}, m_{2}^{t}, \ldots m_{20}^{t}\right\}$ and $M^{c}=\left\{m_{1}^{c}, m_{2}^{c}, \ldots m_{20}^{c}\right\}$, we computed the TPS (Thin Plate Splines) model to obtain the complete correspondence of the 20 minutiae:

$$
F\left(M^{c}\right)=M^{t}
$$

Each minutia is characterised by a triplet $\bar{u}=\{x, y, \vartheta\}$ where $\{x, y\}$ are its Cartesian coordinates in the image and $\vartheta$ is its orientation. The correspondence is defined according to:

$$
F(\bar{u})=c+A \bar{u}+W s(\bar{u})
$$

Where the $c$ and $A$ parameters are referred to the rigid transformation and $W$ indicates the non-linear transformation that includes the elastic deformation, and $s(u)=u^{2} \log u$ is the basis function.

The amount of the deformation is given by a function known as "bending energy": this value can be computed by the elastic parameters $W$ and $s$. Further details for the bending energy computation can be found in [5]. We refer to this feature with the name "SF2".

Moreover, we added some "morphological" features which give a general description of the fingerprint pattern based on its geometrical properties. The first one of these static measures is the mean of the intra-distances among the set of the extracted minutiae (named SF1). For each of these it has been computed the sum of the distances with the other ones. Finally we have considered the mean of this values. The second one is the mean of the ridge width (named SF3). By following the skeleton extracted for each fingerprint images, we computed the value of the width of the correspondent ridge. In fact, the creation of the fake finger (e.g., by the consensual method) includes a sequence of steps which can involve a modification of the width of the ridges and furrows with respect to the correspondent live finger. We give details about the fingerprint reproduction method we followed in Section 3.1.

\subsection{Dynamic Features}

The perspiration is a unique feature of the skin: the perspiration of the pores, which are in every part of the skin, allows to conserve the body temperature into a constant value (body temperature homeostasis). During the contact of a finger on the sensor surface, the perspiration produces a modification of the skin wetness and so of the corresponding image. With a synthetic finger used to spoof a biometric system, the perspiration phenomenon is not present. 
In [3-4] this physiological feature of the skin is used in order to extract some vitality measures from fingerprint images. By using some standard optical and capacitive sensors a time sequence of fingerprint images is captured. The user keeps his fingertip in contact with the surface of the sensor for about 5 seconds, and the sensor captures a certain number of images (frames). The grey-levels variation of two sequential fingerprint frames is a dynamic measure of the perspiration process and, therefore, of the vitality of the finger. These dynamic measures are used as feature vectors submitted to a machine learning algorithm for classification into the "live" and the "fake" fingerprint classes. In order to obtain a good trade-off between the reliability of dynamic features and the usability of the biometric system, an acquisition time of five seconds has been considered [3].

The algorithm for the measure of the perspiration is based on the grey-level values along the ridges path according to the steps described in [4] which we followed:

1) acquisition of two frames of the same fingerprint temporally separated from 5 seconds;

2) separation of the two fingerprint images from the background;

3) binarization and thinning of the last frame;

4) computation of two mono-dimensional signal $\mathrm{C}_{1}$ and $\mathrm{C}_{2}$, containing the grey-level profile along the skeleton extracted at step 3.

The grey-level variations from $\mathrm{C}_{1}$ to $\mathrm{C}_{2}$ represent the dynamic variations of the fingerprint moisture during the acquisition process. Accordingly, a set of dynamic features can be extracted. In [3-4] six measures were computed. However some of these features have not been used in the classification stage. As it is remarked in [3-4], the dynamic measures strongly depend on the acquisition characteristics of the sensor, in particular on the device's dynamic. Therefore, in this work we have selected the following dynamic features from those described in [4]: the time difference of mean grey-level on the skeleton (DF1), the dry saturation percentage change (DF2), and the wet saturation percentage change (DF3). These measures have shown to be appropriate for the capture device we used in our experiments.

Moreover, we added other two dynamic measures: the time variation of the greylevel mean value of the whole image (DF4) and the L1-distance of its grey-levels histogram (DF5).

\section{Experimental Results}

\subsection{The Data Set}

The data-set we used is made up of twenty-eight different fingerprint images from a male population aged between 20 and 40. Images were taken by the Biometrika FX2000 optical sensor.

The data-set is made up of:

- twenty-eight couples of frames (temporally separated from 5 seconds) from live fingerprints;

- twenty-eight couples of frames (temporally separated from 5 seconds) from fake fingerprints of the same subjects;

- twenty-eight additional live impressions of the same subjects. 
The fake data have been created from twenty-eight reproductions of each live fingerprint with the cooperation of the subject, which put his finger on a plasticinelike material. These moulds have been then filled with liquid silicon rubber to create wafer-thin silicon replicas. About one day has been necessary for the solidification of the rubber. Fig. 1 illustrates the main steps of our fingerprint reproduction method.

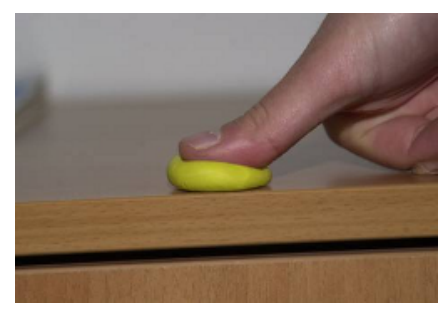

(a)

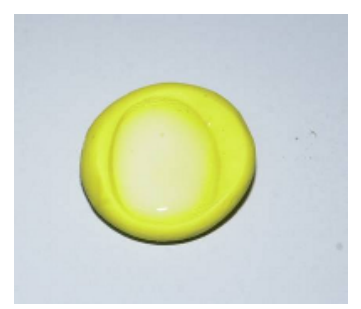

(b)

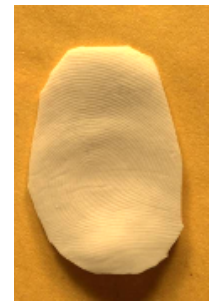

(c)

Fig. 1. Basic steps of our fingerprint reproduction by "consensual" method. (a) The user put his finger on the plasticine-like material, thus creating the mould. (b) The liquid silicon rubber is dripped over the mould. (c) After one day, the solidification of the rubber is completed and it can be removed from the mould. It can be used as a fingerprint stamp.

Our data set has size and characteristics similar to the ones of other data sets used for the vitality detection [3-4]. For example, the data set used in [3] is made of eighteen live finger images, eighteen fake finger images and eighteen finger images from cadavers.

\subsection{Experimental Protocol}

In order to extract the above features from fingerprint images, we adopted the following protocol:

- the second impression has been considered as the template of the fingerprint stored in the system database. The minutiae-points were manually detected in order to avoid errors due to the minutiae detection algorithm;

- the first and the second frame of the first impression have been considered as the images provided by the system during an access attempt. Only attempts related to fingerprints of the same subject were considered ("genuine" attempts by live and fake fingers). Even for these images the minutiae-points were manually detected.

In particular:

- to compute the morphological features (SF1 and SF3), we used the second frame of the first impression;

- to compute the bending energy (SF2), we used twenty-eight images of the second impression as the template of the fingerprint verification system. We computed the bending energy on the basis of the comparison between the template and the second frame of the first impression;

- to compute the dynamic features (from DF1 to DF5) we used the first and the second frame of the first impression;

- each feature was normalised according to: 


$$
f_{i}^{(n)}=\frac{f_{i}-\mu_{i}}{\sigma_{i}}
$$

Where $f_{i}^{(n)}$ is the $i$-th normalised feature $(i=1, \ldots, 8), \mu_{i}$ and $\sigma_{i}$ are the mean and the standard deviation of $f_{i}$ over all available patterns.

Thus, we obtained fifty-six feature vectors made up of three static features from live and fake fingers, and fifty-six feature vector made up of five dynamic features from live and fake fingers.

We used the k-Nearest Neighbour classifier to discriminate between the live and fake fingerprint images characterized by such feature vectors. We performed trials with values of the parameter k between 1 and 27 . For each trial, the accuracy of the kNearest Neighbour classifier was assessed by the leave-one-out method, namely, the accuracy values reported are averaged on fifty-six trials and the $\mathrm{k}$ value corresponding to the highest accuracy is selected.

\subsection{Results}

Table 1 shows the correlation coefficient between features. Some subsets of features (dynamic features especially) exhibit a significant degree of correlation. For example, DF4 and DF5 are negatively correlated with DF1. On the other hand, these features are less correlated with static ones than DF1. Therefore, these results suggest that an appropriate feature selection step is necessary in order to exploit static and dynamic features at best.

Table 2 reports the best performance achieved by using only one feature, in particular the maximum classification accuracy obtained using values of $\mathrm{k}$ ranging in the interval $\{1, \ldots, 27\}$. It is easy to see that static features perform generally worse than dynamic ones, except for SF3.

Table 1. Correlation coefficient of static and dynamic features

\begin{tabular}{|l|r|r|r|r|r|r|r|}
\hline & \multicolumn{1}{c|}{ SF2 } & \multicolumn{1}{c|}{ SF3 } & \multicolumn{1}{c|}{ DF1 } & \multicolumn{1}{c|}{ DF2 } & \multicolumn{1}{c|}{ DF3 } & \multicolumn{1}{c|}{ DF4 } & \multicolumn{1}{c|}{ DF5 } \\
\hline SF1 & 0,08 & 0,29 & 0,36 & 0,15 & 0,31 & $-0,27$ & $-0,27$ \\
\hline SF2 & & 0,09 & 0,19 & $-0,04$ & 0,04 & $-0,16$ & $-0,13$ \\
\hline SF3 & & & 0,66 & 0,68 & 0,04 & $-0,43$ & $-0,43$ \\
\hline DF1 & & & & 0,65 & 0,30 & $-0,85$ & $-0,89$ \\
\hline DF2 & & & & & 0,08 & $-0,47$ & $-0,54$ \\
\hline DF3 & & & & & & $-0,50$ & $-0,24$ \\
\hline DF4 & & & & & & & 0,78 \\
\hline
\end{tabular}

Table 2. Best classification percentage accuracy achieved by the individual features over the investigated values of $\mathrm{k}(\mathrm{k}=1, \ldots, 27)$

\begin{tabular}{l|llllllll} 
& SF1 & SF2 & SF3 & DF1 & DF2 & DF3 & DF4 & DF5 \\
\hline Accuracy (\%) & 53,6 & 57,1 & 62,5 & 85,7 & 60,7 & 82,1 & 75,0 & 71,4
\end{tabular}




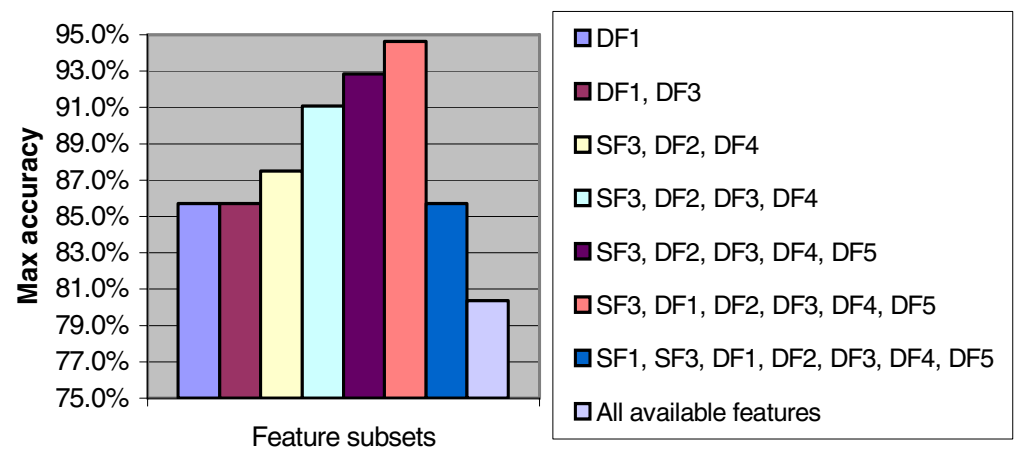

Fig. 2. Overall percentage accuracy over the investigated values of $k(k=1, \ldots, 27)$ for the best subset of each group of $n$ features $(n=1, \ldots, 8)$

In order to detect the best features subsets, we performed classification of fake and live fingers by using the k-NN classifier with all possible subsets of the available features. In other words, we computed the accuracy on the subsets obtained by grouping $\mathrm{n}$ features $(\mathrm{n}=2, \ldots, 8)$. Then we selected the best group of each subset by using the best accuracy over k ranging from 1 to 27 . Fig. 2 shows the overall accuracy of each best group of $n$ features.

Reported results point out that the performance achieved with the best subset is higher than that of the best individual feature (about $10 \%$ more than DF1's accuracy) and that six features allow to obtain the best performance. This subset is made up of SF3 and all the dynamic features (Fig. 2). It is worth noting that the best classification accuracy obtained by using all static features only is $62,5 \%$ and that obtained by using all dynamic features only is $82,1 \%$.

With regard to the best feature subset, it can be noticed that adding the remaining static features negatively affects the performance. However this does not necessarily mean that SF1 and SF2 are not useful for discriminating between live and fake fingers in general, due to the small sample size of the used data set.

Nevertheless, the good performance achieved by combining the dynamic features with the SF3 static feature only can be explained by the physical interpretation that can be associated to these features (especially to dynamic ones [3-4]), which gives more evidence that these results can be expected to hold also on larger data sets. To investigate this hypothesis, let us consider as an example the DF4 and SF3 features.

The first one corresponds to the time difference of the grey-levels mean on the whole images. The expressive power of this feature is evident by looking at Figs. 3(ad), which shows two frames of the same live finger taken at 0 and 5 seconds (Figs. $3(a-b))$, and two sequential frames of the correspondent fake finger (Figs. 3(c-d)). It is evident that the second "live" frame exhibits a higher number of "dark" pixels than that of the first "live" frame, due to the perspiration effect. This does not happen for the two "fake" frames due to the absence of the perspiration phenomenon (see also [3$4]$ ). Therefore, by computing the average difference of the pixels grey-levels between the first and the second frame, we can expect that its value for fake fingers is lower than for live fingers. Similar observations can be made for other dynamic measures. 


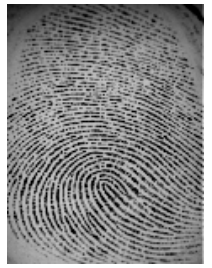

(a)

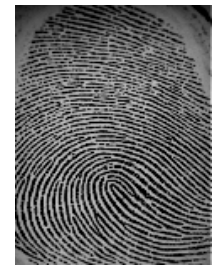

(b)

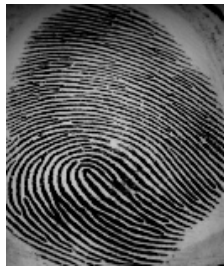

(c)

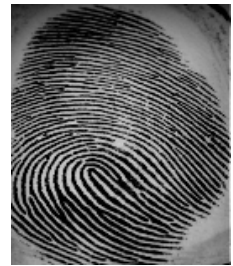

(d)

Fig. 3. (a-b) Two sequential frames of the same live fingerprint acquired by an optical sensor at 0 seconds (a) and 5 seconds (b). (c-d) Two sequential frames of the fake finger correspondent to that of $(a-b)$.

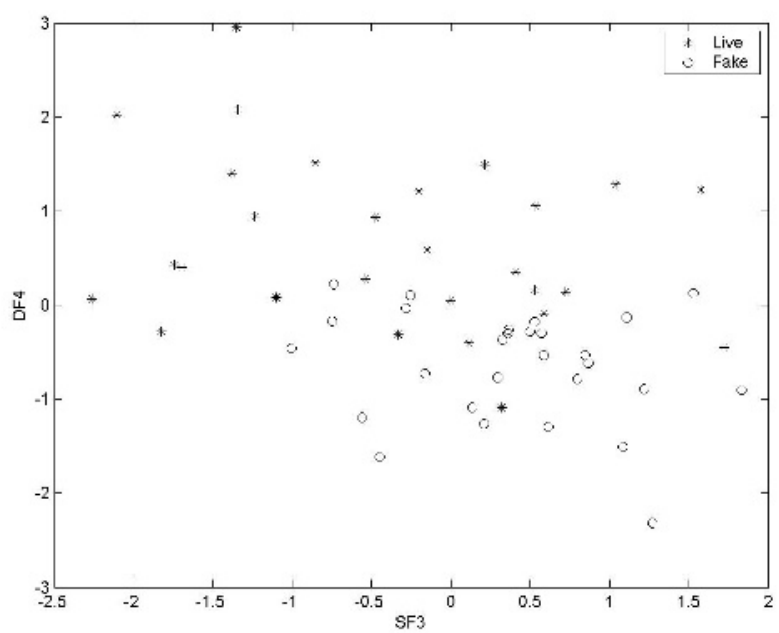

Fig. 4. The patterns of the used data set projected to the SF3-DF4 feature subspace

The second one is the average width of the ridges in a fingerprint image. In fact, the fake fingers creation process involves steps which lead to a different ridges width of the stamp with respect to that of the correspondent live finger (Fig. 1). We observed that the ridges of fake fingerprints images were wider than those of live fingerprints images. Figs. 3(b, d), which is related to a live finger impression and to the correspondent fake finger, clearly show that the ridges of the fake sample are wider than those of the live sample. Therefore, by computing the average width of the fingerprint ridges, we can expect a higher value for fake fingers than for live fingers.

Accordingly, the plot of the pattern projected on the SF3-DF4 feature subspaces should be characterised by fake patterns around low values of the DF4 feature and high values of the SF3 feature and by live patterns spread over the range of each feature, due to the variability of the perspiration phenomenon and the skin characteristics of live fingers each others. Fig. 4 shows the Live and Fake patterns projected to the SF3-DF4 feature subspaces. Distributions of Live and Fake patterns follow the given physical interpretation, thus supporting our hypothesis about the reduction of the impact of the small sample size issue on reported results. 


\section{Conclusions}

In this paper, we investigated static and dynamic features for the vitality detection of fingers. The adopted static features were based on the elastic and morphological properties of the skin, whilst the dynamic ones were based on the perspiration phenomenon as observed in previous works.

So far, no studies on joint static and dynamic features are present in the literature. However, it can be argued that both static and dynamic features could jointly help in distinguishing between live and fake fingers. This preliminary study was aimed to support such hypothesis and also to indicate the most promising of the investigated features. Reported results showed that it was possible to find a subset of static and dynamic features which performed much better than those made up of only one feature or features of the same type. Despite the small size of the used data set, the possibility of associating a physical interpretation to some features suggested that our results could be confirmed even on larger data sets.

\section{References}

1. D. Maltoni, D. Maio, A.K. Jain, S. Prabhakar, Handbook of fingerprint recognition, Springer, 2003.

2. T. Matsumoto, H. Matsumoto, K. Yamada, S. Hoshino, Impact of artificial "gummy" fingers on fingerprint systems, Proc. of SPIE Vol. 4677, Optical Security and Counterfeit Deterrence Techniques IV, pp.24-25, 2002.

3. R. Derakhshani, S. Schuckers, L. Hornak, L. O'Gorman, Determination of vitality from a non-invasive biomedical measurement for use in fingerprint sensors, Pattern Recognition, 36 (2) 383-396, 2003.

4. S. Parthasaradhi, R. Derakhshani, L. Hornak, S. Schuckers, Time-series detection of perspiration as a vitality test in fingerprint devices, IEEE Transactions on Systems, Man and Cybernetics, Part C, 35 (3) 335-343, 2005.

5. Y. Chen, A.K. Jain, S. Dass, Fingerprint deformation for spoof detection, Biometric Symposium, Cristal City, VA, 2005.

6. A. Ross, S. Dass, A.K. Jain, A deformable model for fingerprint matching, Pattern Recognition, 38 95-103, 2005. 